\title{
Polarization controlled femtosecond laser induced birefringence in isotropic crystals
}

\author{
Yuhao Lei, Huijun Wang, Gholamreza Shayeganrad, and Peter G. Kazansky \\ Optoelectronics Research Centre, University of Southampton, Southampton, SO17 1BJ, United Kingdom \\ Yuhao.Lei@soton.ac.uk
}

\begin{abstract}
Polarization controlled birefringent modification by femtosecond laser writing in isotropic crystals is demonstrated. Multiplexed optical data storage with high density in bismuth germanate crystal can be achieved. (C) 2021 The Author(s)

OCIS codes: (260.1440) Birefringence; (320.7090) Ultrafast lasers; (350.3390) Laser material processing
\end{abstract}

\section{Introduction}

Femtosecond (fs) laser writing is a technologically attractive method for birefringence patterning via the construction of nanogratings or elongated nanopores in various bulk and thin film transparent materials. Nanogratings were written by fs laser in crystals, such as tellurium dioxide $\left(\mathrm{TeO}_{2}\right)$ [1], sapphire [2] and quartz [3]. Nanogratings produced polarization dependent birefringence has been reported only in magnesium-oxide-doped lithium niobate $\left(\mathrm{MgO}: \mathrm{LiNbO}_{3}\right)$ crystal along optical axis [4], However, the strong natural birefringence prevents observation of induced birefringence along arbitrary direction, which limits the applications. Here, we demonstrate new type of polarization controlled birefringent modification in the bulk of isotropic crystal, i.e. bismuth germanate $\left(\mathrm{Bi}_{4} \mathrm{Ge}_{3} \mathrm{O}_{12}\right.$, BGO), by fs laser writing and it has been utilized for multidimensional optical data storage.

\section{Results and discussion}

A mode-locked Yb-doped potassium gadolinium tungstate ( $\mathrm{Yb}: \mathrm{KGW})$ regenerative amplified femtosecond laser system (PHAROS, Light Conversion) was employed as a light source operating at a wavelength of $1030 \mathrm{~nm}$ with a pulse duration in the variable range of 190-700 fs. Laser pulses were focused through a $0.50 \mathrm{NA}$ aspheric lens $130 \mu \mathrm{m}$ beneath the surface of a BGO crystal sample (Advatech), which was placed on a three-axial air-bearing translation stage. Laser repetition rate and the sample translation speed varied from $100 \mathrm{kHz}$ to $1 \mathrm{MHz}$ and $0.01 \mathrm{~mm} / \mathrm{s}$ to $40 \mathrm{~mm} / \mathrm{s}$, respectively. The polarization of the writing light was controlled with a Pockels cell and a quarter waveplate.
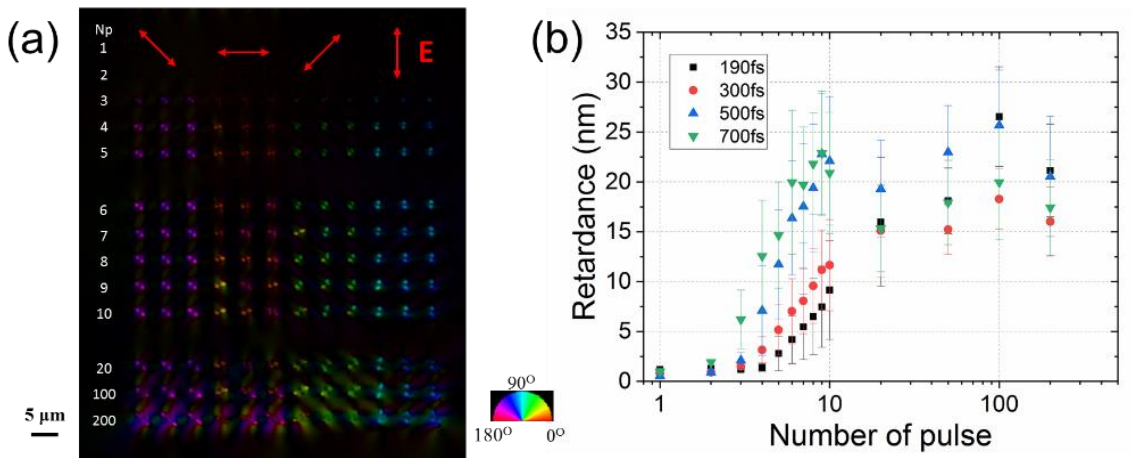

Fig. 1. Birefringent voxels written inside the BGO crystal. (a) Slow axis orientation of written birefringent voxels with a different number of femtosecond pulses $\left(\mathrm{N}_{\mathrm{p}}\right)$. The color represents the orientation of the slow axis and the double arrows show the polarization direction of the writing light. (b) Retardance of the modification as a function of pulse number for different pulse durations from 190 fs to $700 \mathrm{fs}$.

The matrix of birefringent voxels was written in the BGO crystal by focusing femtosecond laser pulses with an energy of $150 \mathrm{~nJ}$ and a pulse duration of $300 \mathrm{fs}$ [Fig. 1(a)]. For the birefringent modifications, the orientation of the slow axis was parallel to the polarization of the writing laser beam, which is different from other materials like silica glass. In addition, the birefringent structure in the BGO crystal has dumbbell shape along the polarization of writing laser light, diverse from the shape in silica glass as well. The different azimuth of slow axis and shape suggest the new formation mechanism of the dumbbell shape birefringent modification compared with the fs laser induced nanogratings or nanopores in silica glass. For pulse number smaller than two, no birefringent modification was 
observed. The retardance of birefringent structures growths rapidly for pulse numbers less than 10 and then followed by a plateau-like behaviour as pulse number increases [Fig. 1(b)]. In addition, the retardance of birefringent modification increases with the pulse duration as pulse number was less than 10 .
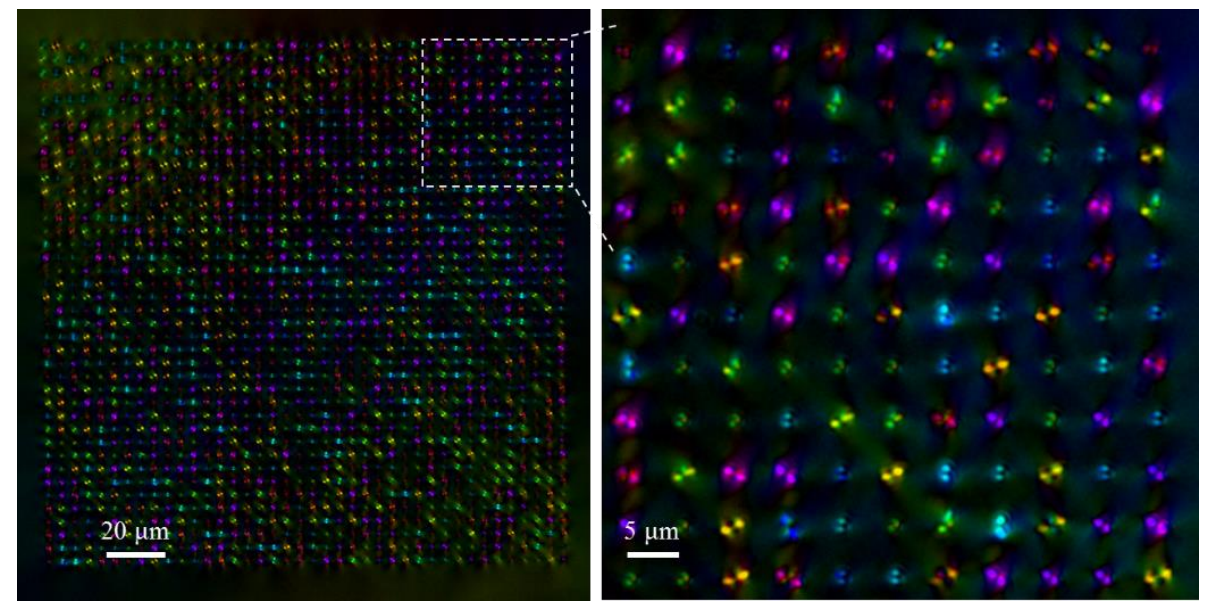

Fig. 2. Birefringent image of imprinted voxels in the BGO crystal written in two retardance levels. The structure was induced at a fixed $150 \mathrm{~nJ}$ pulse energy, $700 \mathrm{fs}$ pulse duration, $1 \mathrm{MHz}$ repetition rate, $1030 \mathrm{~nm}$ wavelength, and focusing via a 0.5 NA lens.

Multiplexed optical data storage has been implemented by femtosecond lasers nanostructuring of fused quartz with advantages of high capacity and long life time [5]. Here, we demonstrated 3 bits/voxel data storage in the BGO crystal by encoding four azimuth angles and two retardance levels in each data voxel [Fig. 2]. It is noticeable that the pulse number for two distinguishable retardance levels were 10 and 4, respectively. Therefore, the data recording speed would be higher than previous 5D optical storage in silica glass by nanogratings or elongated nanopores, which normally requires about one hundred pulses per voxel. Moreover, apart from the dimensions of retardance and azimuth of the slow axis, we could encode additional dimension in the spatial shape of dumbbell structures for multiplexed optical data storage with higher capacity.

\section{Conclusion}

We demonstrated polarization controlled birefringent modification in the bulk of isotropic BGO crystal by fs laser writing, which has a different formation mechanism compared with nanogratings or elongated nanopores induced in silica glass. Multiplexed optical data storage was realized in the BGO crystal with a few fs pulses per voxel to achieve high data writing speed. To realize higher data capacity, the extra dimension than 5D could be further encoded by controlling the spatial shape of the dumbbell birefringent modification.

\section{References}

[1] Y. Shimotsuma et al., "Nano-modification inside transparent materials by femtosecond laser single beam." Mod. Phys. Lett. B 19, 225 (2005).

[2] D. Wortmann et al., "Micro-and nanostructures inside sapphire by fs-laser irradiation and selective etching." Opt. Express, 16, 1517 (2008).

[3] F. Zhang et al., "Self-assembled three-dimensional periodic micro-nano structures in bulk quartz crystal induced by femtosecond laser pulses." Opt. Express, 27, 6442 (2019).

[4] P. Karpinski et al., "Engineering optical anisotropy in nonlinear crystals with ultrafast light." J. Appl. Phys. 15, 153104 (2020).

[5] J. Zhang et al., "Seemingly unlimited lifetime data storage in nanostructured glass." Phys. Rev. Lett. 112, 033901 (2014). 